\title{
Emergence of stable two-colour states in mutually delay-coupled lasers
}

\author{
Masoud Seifikar ${ }^{1,2, \star}$, Andreas Amann ${ }^{1,3, \star \star}$, and Frank H. Peters ${ }^{1,2, \star \star \star}$ \\ ${ }^{1}$ Tyndall National Institute, Lee Maltings, Dyke Parade, Cork T12 R5CP, Ireland \\ ${ }^{2}$ Department of Physics, University College Cork, Cork, Ireland \\ ${ }^{3}$ School of Mathematical Sciences, University College Cork, Cork T12 XF62, Ireland
}

\begin{abstract}
We theoretically investigate a setup of two mutually delay-coupled semiconductor lasers in a face to face configuration, and study the multi-stabilities and symmetry-broken one-colour and two-colour states for this system, for the development of mutually coupled lasers for integration in a Photonic Integrated Circuit (PIC). We show that the stable two-colour exist for the finite distance between lasers.
\end{abstract}

\section{Introduction}

A system of two mutually coupled semiconductor lasers has been widely studied, both experimentally and theoretically $[1,2]$. This system is attracting interest for its potential performance in future all-optical signal processing.

In this paper the physics of mutually injection locked lasers within a photonic integrated circuit (PIC) will be investigated. It is of importance in the creation of advanced modulation formats within a PIC and also has additional academic interest in terms of laser dynamics.

The lasers are coherently coupled via their optical fields, where the delay time $\tau$ arises from the finite propagation time of the light from one laser to the other. This system, which is illustrated in Fig. 1, is well described by single mode rate equations, which are a system of delay differential equations (DDEs) with one fixed delay. Yanchuk et al. [3] predicted one-colour symmetric states for very small delays. Later Erzgräber et al. [4, 5] studied the bifurcations of one-colour states for large delay. Moreover, for zero delay, stable symmetric and symmetry-broken one-colour and two-colour states have been recently predicted by Clerkin et al. [6]. In particular, symmetry-broken two-colour states are highly interesting from an application point of view, for example in the context of all-optical switching [6].

investigate the bifurcation and stability of this system for a finite delay time around $\tau=1$ (in units of photon lifetime), which means the separation around $1.5 \mathrm{~mm}$ for our lasers. Here we show that stable two-colour states continue to exist for finite delays. We perform a detailed study of the relevant bifurcations of the system using the continuation Matlab package DDE-BIFTOOL [7]. As we are interested in the coupling between lasers in a PIC, in this paper we

\footnotetext{
^e-mail: masoud.seifikar@tyndall.ie

$\star \star$ e-mail: a.amann@ucc.ie

$\star \star \star$ e-mail: frank.peters@tyndall.ie
}

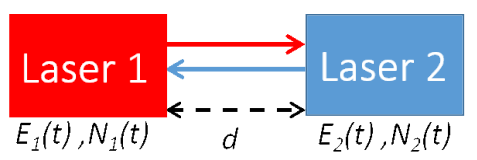

Figure 1. Schematic of two semiconductor lasers, which are mutually coupled by their optical fields.

We present in the next section an overview of the rate equation model that we use to study the physics of the system of mutually coupled lasers. We then solve these DDEs numerically for a given delay, coupling strength and coupling phase in $\S 3$, when we see symmetric and symmetrybroken one-colour and two-colour states for different input parameters. Then in order to understand the origin of these states, we use DDE-BIFTOOL to study the relevant bifurcations of the system with finite delays in $\S 4$, where we first introduce the compound laser modes (CLMs), and explain how CLMs can be continued numerically. We then present the results for symmetric and symmetry-broken states in $\S 4.1$ and $\S 4.2$, respectively. Finally we summarize our conclusion in $\S 5$.

\section{Rate equation model}

We consider two identical semiconductor lasers in a face to face configuration, as illustrated in Fig. 1. We employ the Lang-Kobayashi-type rate equation to model the two mutually coupled lasers. We write the equation for the normalized complex slowly varying envelope of the optical fields $E_{1}$ and $E_{2}$, where $E_{1,2}=\mathfrak{R}\left[E_{1,2}\right]+\mathfrak{J}\left[E_{1,2}\right]$, and the normalized inversions $N_{1}$ and $N_{2}$, for laser 1 and laser 2 , respectively as [5]

$$
\begin{aligned}
& \frac{\mathrm{d} E_{1}(t)}{\mathrm{d} t}=(1+i \alpha) N_{1}(t) E_{1}(t)+\kappa e^{-i C_{p}} E_{2}(t-\tau)-i \Delta_{1}(t), \\
& \frac{\mathrm{d} E_{2}(t)}{\mathrm{d} t}=(1+i \alpha) N_{2}(t) E_{2}(t)+\kappa e^{-i C_{p}} E_{1}(t-\tau)-i \Delta_{2}(t)
\end{aligned}
$$




$$
\begin{aligned}
& T \frac{\mathrm{d} N_{1}(t)}{\mathrm{d} t}=P-N_{1}(t)-\left(1+2 N_{1}(t)\right)\left|E_{1}(t)\right|^{2}, \\
& T \frac{\mathrm{d} N_{2}(t)}{\mathrm{d} t}=P-N_{2}(t)-\left(1+2 N_{2}(t)\right)\left|E_{2}(t)\right|^{2},
\end{aligned}
$$

Here, time $t$ is measured in the units of photon lifetime, $\tau_{p} . T, \alpha$ and $P$ are the parameters of the Laser. $T=392$ is the normalized carrier lifetime, calculated by the ratio of the photonic and carrier lifetime. $\alpha=2.6$ is the linewidth enhancement factor, and $P=0.23$ is the pumping parameters, which describes the amount of the electrical current used to pump the electron-hole pairs in each lasers. In this paper we concentrate on the case with zero detuning $\Delta=0$.

Equations 1-4 are given in the reference frame of rotation with the averaged optical angular frequency of the two lasers $\Omega_{0}=\Omega_{1}+\Omega_{2}$, where $\Omega_{1}$ and $\Omega_{2}$ are the optical angular frequencies of Laser 1 and 2 , respectively. Therefore the optical fields of laser 1 and 2 are given by $E_{1}(t) \exp \left(i \Omega_{0} t\right)$ and $E_{2}(t) \exp \left(i \Omega_{0} t\right)$.

The time delay comes from the distance between two lasers, $\tau=d / c$, where $d$ is the separation of lasers and $c$ denotes the speed of light. As we are interested in the coupling between lasers in a PIC, our main focus is on the small separation around $1 \mathrm{~mm}$. Our main bifurcation parameters are coupling rate $\kappa$ and coupling phase $C_{p}$. Coupling phase can be engineered by changing the distance between lasers as: $C_{p}=2 * \pi\left(d \bmod \lambda_{0}\right)$. In the reference frame of rate equations $1-4, C_{p}$ is changing with optical frequency as $C_{p}=\omega_{0} \tau$. Thus, $C_{p}$ can be fine-tuned by exploiting the temperature or pump current dependency of $\omega_{0}$. However, to allow the change interference condition between the optical fields of the lasers, we consider the coupling phase as an independent parameter.

Equations 1-4 are the system of DDEs with a single fix delay, with number of symmetries; $S^{1}$-symmetry which is a phase space symmetry [5]. It means that any solution of the rate Eqs. 1-4 is invariant under any phase shift of both electric fields $E_{1} \rightarrow E_{1} e^{i b}$. Moreover, the system has the $\pi$-translational and $2 \pi$-translational symmetries. The first one means that $\pi$ shift in coupling phase $C_{p}$ is equivalent to a sign change in the optical field of one laser. The second one means that a $2 \pi$ shift in the phase shift leaves the system unchanged. This means that phase is changing in a circle.

In the rest of this paper we first solve the rate equations 1-4 for given $\tau, \kappa$ and $C_{p}$, in $\S 3$. The calculated optical fields frequencies indicates the existence of onecolour and two-colour, symmetric and symmetry-broken states for different values of parameters. In $\S 4$ we then find a CLM of the laser and continue that solution using the continuation software package DDE-BIFTOOL, which helps us to understand the behaviour of the mutually coupled lasers, and predict the stability regions for wide range of given parameters.

\section{Solution of the DDEs}

In order to solve Eqs. 1-4 we first separate the real and imaginary parts of $E_{1,2}$, which gives a system of 6 DDEs
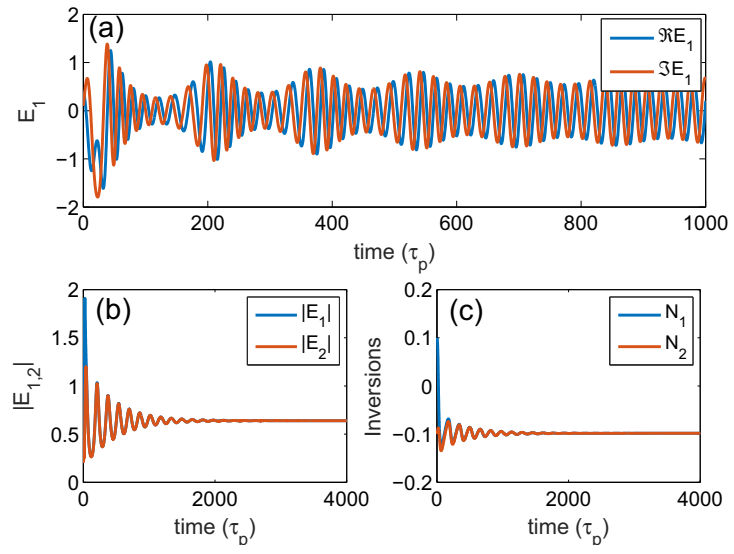

Figure 2. (a) The real and imaginary part of the optical field of the Laser 1. (b) the absolute value of the optical fields and (b) the inversion of Laser 1 and 2, for $\tau=0.5, \kappa=0.1$ and $C_{p}=0.1 \pi$.

with a constant delay $\tau$

$$
\begin{aligned}
\frac{d \mathfrak{R}\left(E_{1}(t)\right)}{d t}= & N_{1}(t) \mathfrak{R}\left(E_{1}(t)\right)-\alpha N_{1}(t) \mathfrak{J}\left(E_{1}(t)\right)+\kappa \cos \left(C_{p}\right) \\
& \times \mathfrak{R}\left(E_{2}(t-\tau)\right)+\kappa \sin \left(C_{p}\right) \mathfrak{J}\left(E_{2}(t-\tau)\right), \\
\frac{d \mathfrak{R}\left(E_{2}(t)\right)}{d t}= & \alpha N_{1}(t) \mathfrak{R}\left(E_{1}(t)\right)+N_{1}(t) \mathfrak{J}\left(E_{1}(t)\right)+\kappa \cos \left(C_{p}\right) \\
& \times \mathfrak{J}\left(E_{2}(t-\tau)\right)-\kappa \sin \left(C_{p}\right) \mathfrak{R}\left(E_{2}(t-\tau)\right), \\
\frac{d \mathfrak{R}\left(E_{1}(t)\right)}{d t}= & N_{2}(t) \mathfrak{R}\left(E_{2}(t)\right)-\alpha N_{2}(t) \mathfrak{J}\left(E_{2}(t)\right)+\kappa \cos \left(C_{p}\right) \\
& \times \mathfrak{R}\left(E_{1}(t-\tau)\right)+\kappa \sin \left(C_{p}\right) \mathfrak{J}\left(E_{1}(t-\tau)\right), \\
\frac{d \mathfrak{J}\left(E_{2}(t)\right)}{d t}= & \alpha N_{2}(t) \mathfrak{R}\left(E_{2}(t)\right)+N_{2}(t) \mathfrak{J}\left(E_{2}(t)\right)+\kappa \cos \left(C_{p}\right) \\
& \times \mathfrak{J}\left(E_{1}(t-\tau)\right)-\kappa \sin \left(C_{p}\right) \mathfrak{R}\left(E_{1}(t-\tau)\right), \\
& \frac{P}{T}-\frac{N_{1}(t)}{T} \\
& -\frac{1+2 N_{1}(t)}{T}\left(\left[\mathfrak{R}\left(E_{1}(t)\right)\right]^{2}+\left[\mathfrak{J}\left(E_{1}(t)\right)\right]^{2}\right), \\
\frac{d N_{1}(t)}{d t}= & \frac{P}{T}-\frac{N_{2}(t)}{T} \\
\frac{d N_{2}(t)}{d t}=2 N_{2}(t) & \left.\left.T \mathfrak{R}\left(E_{2}(t)\right)\right]^{2}+\left[\mathfrak{J}\left(E_{2}(t)\right)\right]^{2}\right),
\end{aligned}
$$

Here we have assumed $\Delta=0$. For given parameters $\kappa$ and $C_{p}$ we solve these system of DDEs numerically using Matlab code based on explicit Runge-Kutta method and calculate the optical fields $E_{1,2}$ and inversions $N_{1,2}$. For instance, Fig. 2 displays optical fields and inversions for $\tau=0.5, \kappa=0.1$ and $C_{p}=0.1 \pi$.

The time traces of real and imaginary parts of the optical field of Laser 1 is shown Fig. 2.a. Also Figs. 2.b and c indicate that for specific parameters, optical fields and inversions of both lasers converge to constant values after 


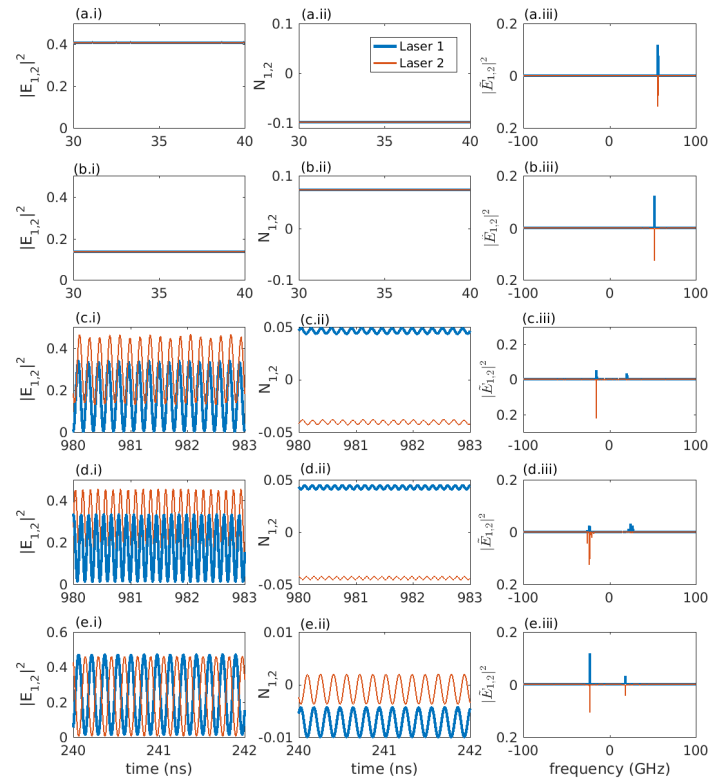

Figure 3. Time trace (left panels), Inversions (middle panels) and frequency spectra (right panels) of Laser 1 and 2, for $\tau=0.5, \kappa=$ 0.1 and $C_{p}=0.1 \pi$ (a panels), $C_{p}=0.2 \pi$ (b and c panels), $C_{p}=$ $0.3 \pi$ (d panels) and $C_{p}=0.513 \pi$ (e panels). Initial conditions for panel a,b, d and e: $\mathfrak{R}\left(E_{1}(t=0)\right)=0.4, \mathfrak{J}\left(E_{1}(t=0)\right)=0$, $\mathfrak{R}\left(E_{2}(t=0)\right)=-0.4, \mathfrak{J}\left(E_{2}(t=0)\right)=0, N_{1}(t=0)=0.05$ and $N_{2}(t=0)=0.05$, for panel $\mathrm{c}$ we only changed $\mathfrak{R}\left(E_{2}(t=0)\right)=$ -0.39 , and $N_{2}(t=0)=-0.05$.

about $2000 \tau_{p}$. In these plots time is given in the unit of photon lifetime $\tau_{p}$, which expresses the rate at which photons are lost from the cavity. For a laser with the cavity length $L$ and mirror reflectivity $R_{1}$ and $R_{2}$ we can estimate the photon life time by

$$
\tau_{p}=-\frac{2 n L}{c} \frac{1}{\operatorname{Ln}\left(R_{1} R_{2}\right)}
$$

where $n$ is the refractive index, and $c$ is the light velocity. For instance for the laser cavity length $L=1.1 \mathrm{~mm}$, with the low reflectivities $R_{1}=R_{2}=0.1$, the photon lifetime is about $\tau_{p}=4.9 \mathrm{ps}$.

Having the slowly varying optical fields of the lasers we can find the rapidly oscillating physical fields $\tilde{E}_{1}$ and $\tilde{E_{2}}$ by taking the Fourier transform of the time trace of the optical fields [6]. Fig. 3 demonstrates the changes in optical field, inversion and frequency of the lasers by changing coupling phase. The time trace of the absolute value of optical fields for $\tau=0.5, \kappa=0.1$ and several values of $C_{p}$ are shown in Figs. 3 (a-e).i (the left column panels), and the middle panels (Figs 3 (a-e).ii) display the inversion for given parameters. The frequency spectra of lasers (relative to the optical frequency of the free running lasers) are given in the right hand side plots (Figs 3 (a-e).iii).

In Figs 3.a, we observe a symmetric one-colour state for $\tau=0.5, \kappa=0.1$ and $C_{p}=0.1 \pi$, as the lasers have the same optical fields and inversions, and are lasing with a common locked frequency around $56 \mathrm{GHz}$. By increas-



Figure 4. Pitchfork (solid lines) and Hopf (dashed lines) bifurcation diagram of system for $\tau=0.01-2$.

ing phase to $C_{p}=0.2 \pi$, we can obtain either symmetric one-colour (Figs 3.b) or symmetry-broken two-colour (Figs 3.c), depending on the initial conditions that is used to solve the DDEs in Eqs. 1-4. However, if we increase phase further to $C_{p}=0.3 \pi$ we can get only symmetrybroken two-colour stable states, as shown in Figs 3.d. For a very small window of $C_{p}$ we are able to get symmetric two-colour states as well. For instance, Figs. 3.e display such a states for $C_{p}=0.513 \pi$.

In order to predict the stability of the states for a given parameters, we need to calculate the bifurcation diagram using the DDEs given in Eqs. 1-4. In the next section we use MATLAB package DDE-BIFTOOL [7], which allows us to find the stability information, and bifurcations by following equilibria and periodic orbits.

\section{Bifurcation diagrams}

Here, we perform a detailed study of the relevant bifurcations of the system using the continuation Matlab package DDE-BIFTOOL. The basic solutions of the system, for a symmetric one-colour state, are given by [5]

$$
\begin{array}{r}
E_{1}(t)=R_{1}^{s} e^{i \omega_{s} t}, \\
E_{2}(t)=R_{1}^{s} e^{i \omega_{s} t+i \sigma}, \\
N_{1}(t)=N_{1}^{s}, \\
N_{2}(t)=N_{2}^{s}
\end{array}
$$

These solutions are called the compound laser modes (CLMs). Here $R_{1,2}^{s}, N_{1,2}^{s}, \omega_{s}$, and $\sigma$ are real and timeindependent values. We assumed lasers have the same frequency $\omega_{s}$, which is the deviation between the average solitary laser frequency $\Omega_{0}$ and the frequency of the coupled laser system. The relationship between the frequency $\omega_{s}$ and the phase difference $\sigma$, can be obtained by inserting the ansatz 7 into rate equations 1-4, and eliminating the unknown variables $R_{1}, R_{2}, N_{1}, N_{2}$, which gives:

$$
\omega_{s}= \pm \kappa \sqrt{1+\alpha^{2}} \sin \left(C_{p}+\omega_{s} \tau+\arctan (\alpha)\right)
$$



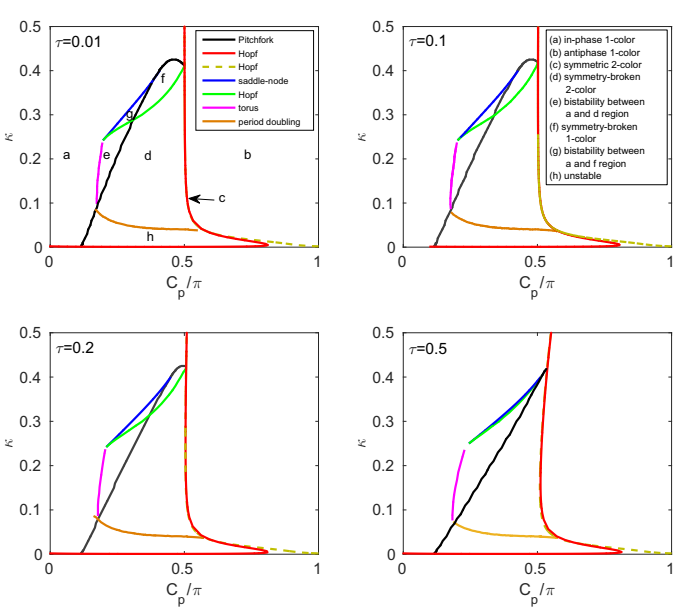

Figure 5. Bifurcations and stability regions in coupling strength $\kappa$ and coupling phase $C_{p}$ surface for $\tau=0.01,0.1,0.2$ and 0.5 .

Here + and - are corresponding to the in-phase $(\sigma=0)$ and the anti-phase $(\sigma=\pi)$ CLMs, respectively. For a given delay time, we start with the basic solution of the system, given by Eq. 8 and then continue this solution to find bifurcations and stabilities for two bifurcation parameters of coupling phase $C_{p}$ and coupling strength $\kappa$.

\subsection{Symmetric solutions}

The solution of system can be obtained by the DDEBIFTOOL, which gives the stability information and bifurcation along each branch. The dashed lines in Fig. 4 show the Hopf lines that are obtained by continuation of the initial Hopf point. The solid lines in Fig. 4 display the pitchfork bifurcations, for delay times $\tau=0.01-2$. The blue curves in this plot show the result for very small delay time $(\tau=0.01)$, which is similar to what Yanchuk [3] has already obtained analytically for $\tau=0$. The area on the left hand side of the pitchfork line, with the low coupling phase, gives stable in-phase one-colour states, while the region on the right hand side of the Hopf lines, is antiphase one colour states. At very high coupling strengths, these regions are separated by two supercritical Hopf lines in close proximity. There is no stable symmetric state in the area between Hopf and pitchfork lines, However as we will see in the next section, there are stable symmetrybroken states in this region. Fig. 4 shows that the slope of pitchfork line decreases with increasing delay time $\tau$. The pitchfork-Hopf point is moving to lower $\kappa$ and higher $C_{p}$ by increasing the delay time.

\subsection{Symmetry-broken solutions}

The bifurcation diagram shown in Fig. 4 provides the stability information only for the symmetric states. However using the DDE-BIFTOOL we are able to calculate the symmetry-broken bifurcations as shown in Fig. 5 [4]. Bifurcations and stability regions with the two bifurcation parameters, coupling strength $\kappa$ and coupling phase $C_{p}$ are


Figure 6. The frequency spectrum relative to the frequency of the free-running lasers, for $\tau=0.01, \kappa=0.33$ and $C_{p}=0.35 \pi$ (a and b) and $C_{p}=0.35 \pi(\mathrm{c})$. Initial conditions for panel a and c: $\mathfrak{R}\left(E_{1}(t=0)\right)=0.4, \mathfrak{J}\left(E_{1}(t=0)\right)=0, \mathfrak{R}\left(E_{2}(t=0)\right)=-0.4$, $\mathfrak{J}\left(E_{2}(t=0)\right)=0, N_{1}(t=0)=0.05$ and $N_{2}(t=0)=0.05$, for panel $\mathrm{b}$ we only changed $\mathfrak{R}\left(E_{2}(t=0)\right)=-0.39$, and $N_{2}(t=0)=$ -0.05 .

shown in Fig. 5 for $\tau=0.01,0.1,0.2$ and 0.5. The symmetric pitchfork and Hopf bifurcations are shown by black, red and dashed yellow lines. Two Hopf lines are very close for $\kappa>0.05$, however there is a tiny gap between them which gets wider by increasing delay time, $\tau$. The blue and green curves in Fig. 5 display symmetric-broken saddle-node and Hopf lines, pink line shows the torus bifurcation, and orange line represents the period-doubling bifurcation. The plot for $\tau=0.01$, shown on the top left panel of this figure is very similar to what Clerkin et al. [6] obtained for zero delay time, using the continuation software AUTO [8].

The bifurcation diagrams in Fig. 5 confirm that we can have stable two-colour states for finite delay time as well. In region $\mathrm{d}$ which is confined by pitchfork and Hopf lines at the sides and another Hopf and period-doubling lines at the top and bottom, we observe stable symmetric-broken two-colour states. In region e we can get either symmetric one-colour or symmetry-broken two-colour states as already shown in Figs. 3.b and c. In the tiny region between two Hopf lines (c), we can get symmetric twocolour states as was displayed in Figs. 3.e. Moreover, in region f symmetry-broken one-colour states are stable. In region $g$ we might get symmetric or symmetry-broken one-colour states depending on the initial conditions. This is consistence with Fig. 6 , for $\tau=0.1$ and $\kappa=0.33$. The corresponding points in Fig. 6.a and b, with $C_{p}=0.35 \pi$ are located in region g, while Fig. 6.c with $C_{p}=0.38 \pi$ is located in region $\mathrm{f}$. Fig. 5 indicates that regions $\mathrm{f}$ and $\mathrm{g}$ are shrinking by increasing the distance between lasers and disappear for $\tau$ larger than 0.5 .

As shown in Fig. 7, for $\tau=1$, the torus bifurcation continues along the pitchfork line to reach the Hopf lines. 


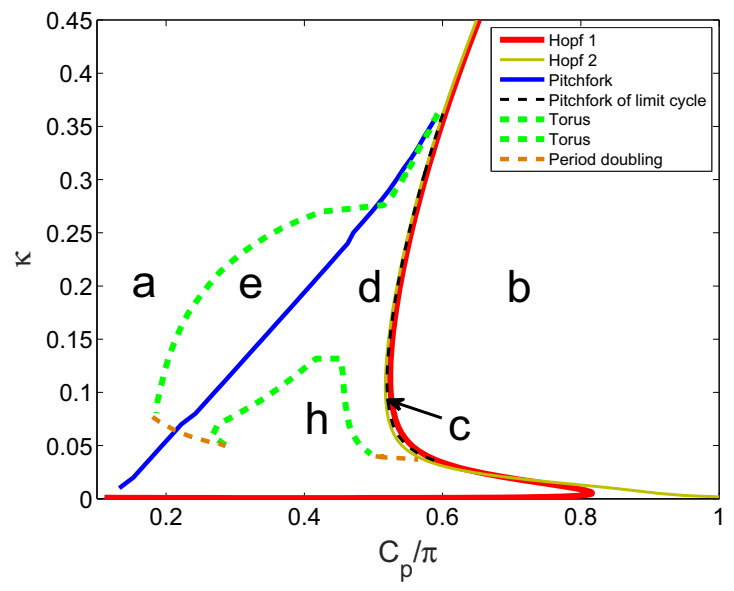

Figure 7. Bifurcations and stability regions in coupling strength $\kappa$ and coupling phase $C_{p}$ plane, for delay time $\tau=1$. Solid lines display the symmetric Hopf and pitchfork bifurcations. The dashed curves show the bifurcations of the limit cycles.Stable symmetric-broken two-coulor states observed for intermediate coupling strength and phase.

Another branch of torus bifurcation emerges at the lower values of coupling which makes the middle part of the period doubling line unstable. Similar to what we have already observed for the lower values of delay time, in regions $a$ and $b$, in-phase and antiphase one-colour states are stable, respectively. The very small area between two Hopf lines contains region c with stable symmetric twocolour states. More importantly, the model predicts that symmetry-broken two-colour states are stable in the two large regions $\mathrm{d}$ and $\mathrm{e}$, which are bounded by torus, perioddoubling and pitchfork of limit cycle bifurcations. Region e corresponds to the area of bistability between symmetric one-colour (region a) and symmetry-broken two-colour states (region d).

\section{Discussion and conclusions}

In summary we theoretically investigated a setup of two mutually delay-coupled semiconductor lasers in a face to face configuration, and studied the multi-stabilities and symmetric and symmetry-broken one-colour and twocolour states for this system. We showed that the stable symmetry-broken two-colour states continue to exist for finite delay time, and therefore stable states exist for almost all values of coupling phase $C_{p}$ and delay time up to 1. However, increasing delay time makes the area with intermediate $C_{p}$ and low value of $\kappa$ unstable. The presented results indicate that while the overall shape of the bifurcation diagram changes rapidly around $\tau=1$, the stable two-colour states still can be achieved for a wide range of coupling strength $\kappa$ and coupling phase $C_{p}$.

Acknowledgments We thank Bernd Krauskopf and Hartmut Erzgräber for helpful discussions and for help with the DDE-BIFTOOL implementation. This work was supported by the Science Foundation Ireland under grant SFI 13/IA/1960.

\section{References}

[1] H.G. Schuster, K. Lüdge, Nonlinear laser dynamics: from quantum dots to cryptography (John Wiley \& Sons, 2012)

[2] M.C. Soriano, J. García-Ojalvo, C.R. Mirasso, I. Fischer, Rev. Mod. Phys. 85, 421 (2013)

[3] S. Yanchuk, L. Recke, Physical Review E 69, 056221 (2004)

[4] H. Erzgräber, D. Lenstra, B. Krauskopf, Proceedings Symposium IEEE/IEOS Benelux Chapter, 2004, Ghent pp. 147-150 (2004)

[5] H. Erzgräber, B. Krauskopf, D. Lenstra, SIAM Journal on Applied Dynamical Systems 5, 30 (2006)

[6] E. Clerkin, S. O’Brien, A. Amann, Physical Review E - Statistical, Nonlinear, and Soft Matter Physics (2014)

[7] K. Engelborghs, T. Luzyanina, G. Samaey, TW Report 305 (2000)

[8] E.J. Doedel, T.F. Fairgrieve, B. Sandstede, A.R. Champneys, Y.A. Kuznetsov, X. Wang (2007) 